\title{
Using a respiratory navigator significantly reduces variability when quantifying left ventricular torsion with cardiovascular magnetic resonance
}

Sean M. Hamlet 1,2, Christopher M. Haggerty ${ }^{2,3,4}$, Jonathan D. Suever ${ }^{2,3,4}$, Gregory J. Wehner ${ }^{2,5}$, Kristin N. Andres $^{2}$, David K. Powell ${ }^{5}$, Richard J. Charnigo ${ }^{6}$ and Brandon K. Fornwalt $2,3,4,5,7,8^{*}$

\begin{abstract}
Background: Left ventricular (LV) torsion is an important indicator of cardiac function that is limited by high inter-test variability ( $50 \%$ of the mean value). We hypothesized that this high inter-test variability is partly due to inconsistent breath-hold positions during serial image acquisitions, which could be significantly improved by using a respiratory navigator for cardiovascular magnetic resonance (CMR) based quantification of LV torsion.
\end{abstract}

Methods: We assessed respiratory-related variability in measured LV torsion with two distinct experimental protocols. First, 17 volunteers were recruited for CMR with cine displacement encoding with stimulated echoes (DENSE) in which a respiratory navigator was used to measure and then enforce variability in end-expiratory position between all LV basal and apical acquisitions. From these data, we quantified the inter-test variability of torsion in the absence and presence of enforced end-expiratory position variability, which established an upper bound for the expected torsion variability. For the second experiment (in 20 new, healthy volunteers), 10 pairs of cine DENSE basal and apical images were each acquired from consecutive breath-holds and consecutive navigator-gated scans (with a single acceptance position). Inter-test variability of torsion was compared between the breath-hold and navigator-gated scans to quantify the variability due to natural breath-hold variation. To demonstrate the importance of these variability reductions, we quantified the reduction in sample size required to detect a clinically meaningful change in LV torsion with the use of a respiratory navigator.

Results: The mean torsion was $3.4 \pm 0.2 \% \mathrm{~cm}$. From the first experiment, enforced variability in end-expiratory position translated to considerable variability in measured torsion $(0.56 \pm 0.34 \% \mathrm{~cm})$, whereas inter-test variability with consistent end-expiratory position was $57 \%$ lower $(0.24 \pm 0.16 \% \mathrm{~cm}, p<0.001)$. From the second experiment, natural respiratory variability from consecutive breath-holds translated to a variability in torsion of $0.24 \pm 0.10 \% \mathrm{~cm}$, which was significantly higher than the variability from navigator-gated scans $(0.18 \pm 0.06 \% \mathrm{~cm}, p=0.02)$. By using a respiratory navigator with DENSE, theoretical sample sizes were reduced from 66 to 16 and 26 to 15 as calculated from the two experiments.

Conclusions: A substantial portion (22-57\%) of the inter-test variability of LV torsion can be reduced by using a respiratory navigator to ensure a consistent breath-hold position between image acquisitions.

Keywords: Left ventricular torsion, Breath-holds, DENSE, Respiratory navigator gating, Cardiovascular magnetic resonance

\footnotetext{
* Correspondence: bkf@gatech.edu

${ }^{2}$ Department of Pediatrics, University of Kentucky, Lexington, KY, USA

${ }^{3}$ Department of Imaging Science and Innovation, Geisinger Health System,

Danville, PA, USA

Full list of author information is available at the end of the article
} 


\section{Background}

Left ventricular (LV) torsion is an important indicator of cardiac function [1, 2]; however, the quantification of torsion is limited by poor inter-test reproducibility. For example, a previous study with myocardial tagging demonstrated that the inter-test variability of torsion represented nearly $50 \%$ of the mean value [3]. This substantial variability reduces prognostic value for individual patients and leads to larger required sample sizes for research studies to detect meaningful differences or changes. Previous studies have reported that sample sizes ranging from $80-107$ are required to detect a $10 \%$ relative difference in torsion with $90 \%$ power [3-5]. Reducing variability and lowering required sample sizes is important to improve the clinical and research utility of torsion.

LV torsion is typically quantified as the gradient of twist along the longitudinal axis of the heart. This gradient is computed using twist derived from two short-axis images (basal and apical) of the LV and the longitudinal distance between the images [3] (Fig. 1). End-expiratory breath-holds are used to minimize respiratory motion artifacts, and the basal and apical short-axis images are typically acquired during separate breath-holds.

When post-processing the image data to compute LV torsion, the longitudinal distance between the short-axis images is calculated from either A) assumptions derived from an additional longitudinal image (echocardiography) or B) information specifying the location of the imaging planes in $3 \mathrm{D}$ space taken from the Digital Imaging and Communications in Medicine (DICOM) image header (cardiovascular magnetic resonance [CMR]). A confounding factor that is not considered is that the exact endexpiratory position may differ by up to $13 \mathrm{~mm}$ between separate breath-holds [6-10], which creates differences in heart position between the basal and apical image acquisitions (Fig. 2). We hypothesized that inconsistent endexpiratory diaphragm positions during serial breath-holds accounts for a significant portion of the variability in measured LV torsion and that this variability could be reduced by using CMR based quantification of LV torsion with a respiratory navigator.

\section{Methods}

Respiratory-related variability in measured LV torsion was assessed with two distinct experimental protocols: 1) using enforced variability in end-expiratory position between acquisitions and 2) allowing for natural variability in end-expiratory position between acquisitions. The former experiment was performed to establish an upper bound on respiratory-related variability in torsion, while the latter mimics a more relevant clinical setting. In both experiments, the effect of using a respiratory navigator to ensure a consistent end-expiratory position on torsion variability was also quantified. The local Institutional Review Board approved the study protocols, and all subjects provided written informed consent.

\section{LV motion quantification}

Imaging was performed on a 3 Tesla Siemens Tim Trio (Siemens Healthcare, Erlangen, Germany) with a 6element chest coil and a 24-element spine coil. LV twist was measured at basal and apical short-axis locations in both experiments using 2D spiral cine Displacement Encoding with Stimulated Echoes (DENSE) CMR $[11,12]$.

The basal and apical short-axis locations were defined as follows: On a four-chamber image, five short-axis slices were planned equidistant across the end-systolic endocardial ventricular long-axis length. The slices were planned such that the outermost slices did not extend beyond the mitral valve plane and endocardial apex, respectively. The second and fourth slices of this stack were defined as the basal and apical short-axis locations. Imaging parameters were: spiral interleaves $=6$, interleaves per frame $=2, \quad \mathrm{FOV}=360 \times 360 \mathrm{~mm}^{2}$, pixel spacing $=$ $2.8 \times 2.8 \mathrm{~mm}^{2}$, slice thickness $=8 \mathrm{~mm}, \mathrm{TE} / \mathrm{TR}=1.1 / 17 \mathrm{~ms}$,
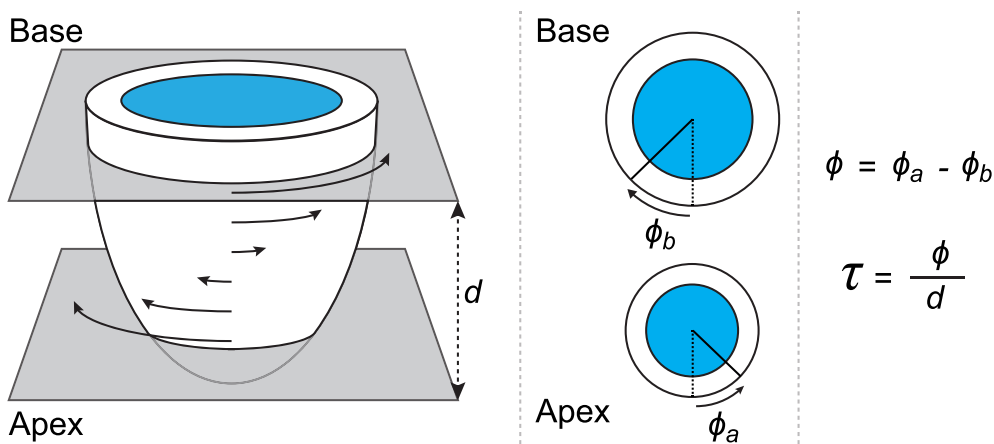

Fig. 1 Computation of LV torsion from basal and apical images. The curved arrows represent the relative twist along the longitudinal axis of the left ventricle. LV twist $(\phi)$ was measured as the difference in rotation between the apex $\left(\phi_{a}\right)$ and base $\left(\phi_{b}\right)$ (twist direction shown as viewed from foot to head). Torsion ( $\tau$ ) was computed as LV twist divided by the distance $(d)$ between basal and apical image locations 


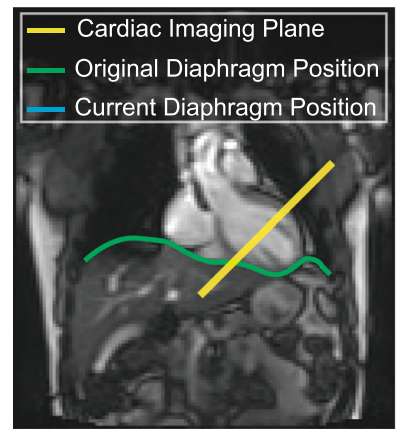

End-Expiration

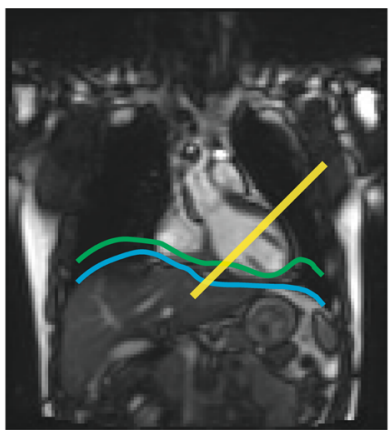

Mid-Inspiration

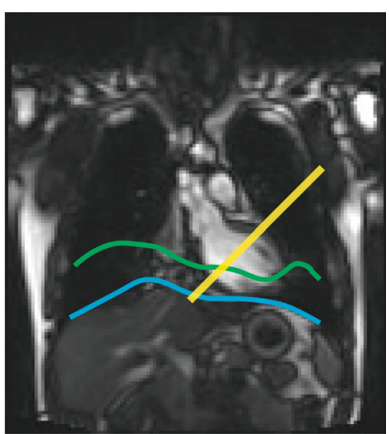

End-Inspiration

Fig. 2 Real-time images of the diaphragm as it translates during a respiratory cycle. During respiration, diaphragm motion causes the heart to translate a substantial distance through the fixed imaging plane

temporal resolution $=34 \mathrm{~ms}$, variable flip angle $=20^{\circ}$, displacement encoding $=0.06 \mathrm{cyc} / \mathrm{mm}$ [13], through-plane dephasing $=0.08 \mathrm{cyc} / \mathrm{mm}$ [14], CSPAMM echo suppression [15], view sharing, prospective ECG gating, and a respiratory navigator with an acceptance window of $\pm 3 \mathrm{~mm}$.

DENSEanalysis [16] was used to derive LV twist from the DENSE images. Epicardial and endocardial contours were manually delineated on the DENSE magnitude images at end-diastolic and end-systolic cardiac phases [17]. Post-processing was performed as previously described [17]. A semi-automatic path-following algorithm was used to unwrap the displacement-encoded phase data. The resulting displacement trajectories were further processed by applying spatial smoothing and temporal fitting [18].

LV twist was computed over the cardiac cycle relative to the centroid of the endocardial boundary at end-diastole. The distance between the basal and apical image locations was calculated from the DICOM headers. LV torsion was computed as the difference in rotation between the apex and base $(\phi)$ divided by the distance $(d)$ between the basal and apical image locations [3, 19, 20] (Fig. 1).

\section{Experiment 1: enforced end-expiratory variability}

Ten healthy volunteers with no known cardiovascular disease or chronic illnesses and seven patients with a history of heart disease (known diagnosis of heart failure, cardiomyopathy, or myocardial infarction) were recruited. We first quantified the end-expiratory variability for each subject by acquiring respiratory navigator measurements (90-180 cross-pair configuration; Fig. 3) of 10 consecutive, 10 -second breath-holds. No cardiac image data were acquired, but the mode position of each breath-hold was retained to identify subject-specific minimum, middle and maximum end-expiratory positions of the diaphragm across the 10 breath-holds (Fig. 4). These subject-specific positions were then used to define the locations of the navigator acceptance windows for subsequent acquisitions of respiratory navigator-gated DENSE. Specifically, the basal and apical slices were both acquired with the navigator acceptance window at each of the three positions. Moreover, the acquisitions at the middle acceptance window location were repeated to define inter-test variability when ensuring a consistent position with a respiratory navigator. For all

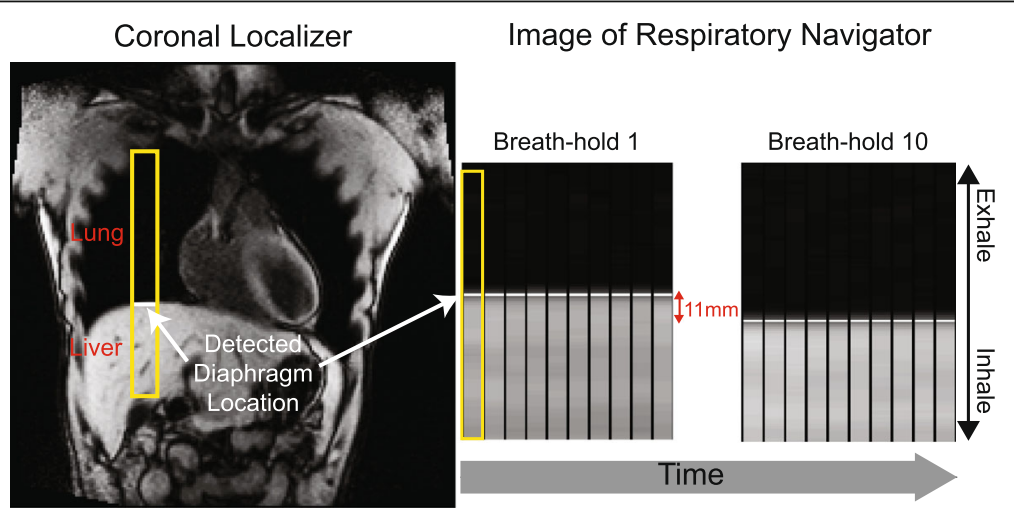

Fig. 3 Respiratory navigator gating. (Left) The diaphragm position is at the high-contrast interface between the lung (dark) and the liver (bright). (Right) Image of a measured diaphragm position over time for separate breath-holds. For this subject, there was an $11 \mathrm{~mm}$ difference in end-expiratory position between breath-hold 1 and 10 


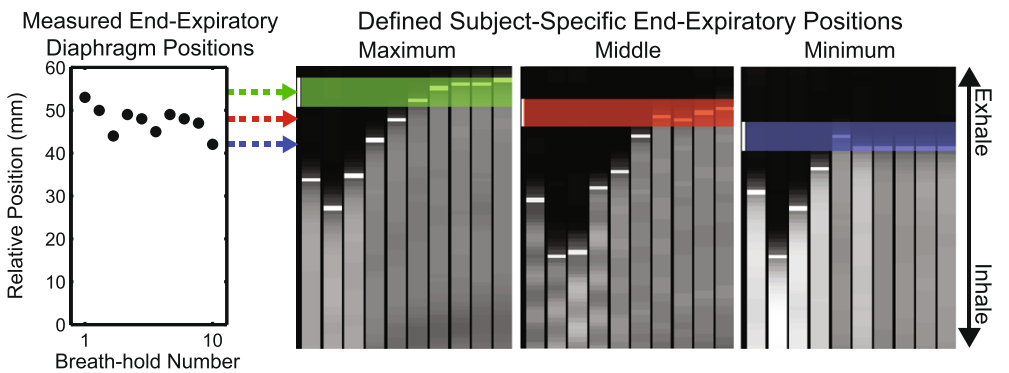

Fig. 4 Measured end-expiratory diaphragm positions were used to define subject-specific maximum, middle, and minimum end-expiratory positions. The maximum diaphragm position was defined as being closer to the end-expiratory position while the minimum diaphragm position was defined as being closer to the end-inspiratory position

scans, the image of the respiratory navigator was projected to the subjects in real-time during DENSE acquisition, which helped to ensure consistent efficiency [21] across scans despite varying acceptance locations.

With three independent measurements at both LV locations, nine permutations of torsion were calculated from the possible combinations (Fig. 5), providing an estimate of torsion variability due to inconsistent endexpiratory positions. This variability in torsion was compared to the inter-test variability (i.e., comparing the two torsion measures acquired at the middle navigator acceptance position, Fig. 5) to isolate respiratory position effects.

\section{Experiment 2: natural end-expiratory variability}

We next sought to quantify the effects of natural endexpiratory variability. Twenty new healthy volunteers were recruited. In these subjects, 10 basal and apical images were each acquired with two protocols: 1) during consecutive breath-holds, and 2) during consecutive navigator-gated acquisitions with a single acceptance window location. In each case, the 10 image pairs were used to derive 20 measurements of LV torsion, by combining each basal twist measurement with the two closest apical twist measurements in the temporal sequence. The torsion variability between these protocols was then quantified to compare the differences as a result of consistent (navigator-gated) and inconsistent (breath-hold) end-expiratory positions. Importantly, to monitor the end-expiratory position of the breath-hold acquisitions, the scans were acquired with the respiratory navigator enabled, but with a wide $( \pm 50 \mathrm{~mm})$ acceptance window width that never resulted in the exclusion of acquired image data.

\section{Statistics}

Statistical analyses were performed using $\mathrm{R}$ version 3.2.2 (R Foundation for Statistical Computing, Vienna, Austria). All continuous variables were expressed as mean \pm standard deviation and group means were compared using student's t-tests. Pearson correlation was used to observe associations between continuous variables.

For experiment 1, the inter-test variability of torsion was quantified using 95\% inter-test limits of agreement of the two middle navigator acceptance window scans. To test for an overall difference in variability between inconsistent and consistent end-expiratory positions, the LV torsion permutations from the variable endexpiratory positions were compared to the $95 \%$ inter-test limits of agreement using a binomial test to evaluate whether values fell within the $95 \%$ limits significantly less than $95 \%$ of the time. The root mean squared error (RMSE) was then computed to quantify the differences in variability. Specifically, the RMSE for the consistent endexpiratory position was computed by computing the mean squared error (MSE) of the two middle acceptance window scans and taking the square root. The RMSE for the LV torsion permutations was computed by separately computing the MSE of the permutations with respect to each of the two middle acceptance window scans, averaging the MSEs, and taking the square root.

\begin{tabular}{|c|c|c|c|c|c|c|c|c|c|c|}
\hline & \multicolumn{9}{|c|}{ Torsion Permutations } & \multirow{2}{*}{$\begin{array}{l}\text { For Inter-test } \\
\text { Comparison } \\
\text { Repeat } 5\end{array}$} \\
\hline & 1 & 2 & 3 & 4 & 5 & 6 & 7 & 8 & 9 & \\
\hline Base & Maximum & Maximum & Maximum & Middle & Middle & Middle & Minimum & Minimum & Minimum & Middle \\
\hline Apex & Maximum & Middle & Minimum & Maximum & Middle & Minimum & Maximum & Middle & Minimum & Middle \\
\hline
\end{tabular}

Fig. 5 The nine possible torsion permutations were constructed from three basal and three apical images. One basal and apical image was acquired for each subject-specific end-expiratory position (maximum, middle, and minimum). Image acquisitions were repeated at the middle position to assess inter-test variability (far right) 
For experiment 2, breath-hold and navigator-gated acquisitions were compared by computing the standard deviations of the 20 respective measurements and performing a Student's t-test. Variability in torsion was also quantified using 95\% inter-test limits of agreement, which were computed using the standard deviation of the difference between consecutive pairs of torsion measurements. For all statistical tests, significance was defined as $p<0.05$.

\section{Theoretical sample size calculation}

To quantify the effects of the differences in torsion measurement variability, we computed theoretical sample sizes required to detect a clinically meaningful change in LV torsion for each experimental condition. Study sample sizes required to detect a $10 \%$ relative difference in LV torsion with a power of $90 \%$ and a significance level of 0.05 were computed using the standard deviation of the inter-test differences in torsion $(\sigma)$ and the equation:

$$
n=f(\alpha, P) \cdot \sigma^{2} \cdot \frac{2}{\delta^{2}}
$$

where $\mathrm{n}$ is the sample size per group, $\alpha$ is the significance level, $P$ is the power, $f$ is the value of the factor for different values of $\alpha$ and $P(f=10.5$ for $\alpha=0.05$ and $P=0.90)$, and $\delta$ is the magnitude of the difference to be detected [22]. To determine the improvement in sample size compared to other modalities, sample sizes calculated by this formula were compared to those calculated based on data from previous studies that quantified LV torsion.

\section{Results}

For experiment 1, ten healthy volunteers (Age: $22 \pm 6$ years, Range: $19-38$ years, $60 \%$ female) and seven patients (Age: $57 \pm 8$ years, Range: $45-67$ years, $43 \%$ female) were enrolled. One healthy volunteer was excluded due to movement during imaging, so data from the remaining nine healthy volunteers are reported. For experiment 2, 20 healthy volunteers (Age: $25 \pm 4$ years, Range: $20-34$ years, $60 \%$ female) were enrolled.

\section{Inconsistent end-expiratory positions}

From experiment 1, the intra-subject range and standard deviation of end-expiratory positions were $10.2 \pm 4.4 \mathrm{~mm}$ and $3.3 \pm 1.4 \mathrm{~mm}$, respectively. There was no significant difference in the range or standard deviation of endexpiratory position between healthy and patient groups $(p=0.94$ and $p=0.70$, respectively; Fig. 6). From experiment 2, the intra-subject range and standard deviation of end-expiratory positions over 20 breath-holds were $13.9 \pm 10.5 \mathrm{~mm}$ and $3.8 \pm 3.1 \mathrm{~mm}$, respectively.

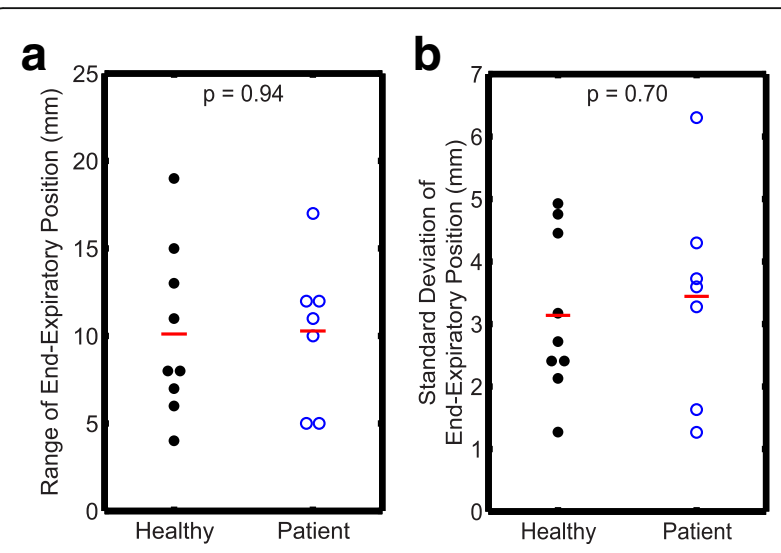

Fig. 6 Inconsistent end-expiratory positions across ten consecutive breath-holds in patients and healthy controls. There were no significant differences in either the range (a) or standard deviation (b) of end-expiratory position between the healthy and patient groups. Solid red lines denote the mean for each group

\section{Torsion}

DENSE images and displacements from a representative subject show the relative twist differences between the base and apex at end-systole (Fig. 7). Table 1 summarizes the LV torsion results for each protocol. From experiment 1, the inter-test limits of agreement at a consistent position were $\pm 0.6 \% \mathrm{~cm}$, and the binomial test indicated that the variability in LV torsion due to enforced variability in end-expiratory position was significantly higher than the variability at a consistent end-expiratory position $(p<0.001)$. Specifically, the RMSE of LV torsion permutations across end-expiratory positions was $0.56 \pm 0.24^{\circ} / \mathrm{cm}$ (range: $0.2-1.3^{\circ} / \mathrm{cm}$ ), while the RMSE from a consistent end-expiratory position was $57 \%$ lower $\left(0.24 \pm 0.16^{\circ} / \mathrm{cm}\right)$. Moreover, there was a moderate correlation across subjects between the torsion RMSE and the range of end-expiratory positions $(\mathrm{r}=0.50, p=0.049$, Fig. 8). Finally, the mean LV torsion for consistent endexpiratory positions was not significantly different between the healthy $\left(3.6 \pm 1.2^{\circ} / \mathrm{cm}\right)$ and patient $\left(3.2 \pm 1.3^{\circ} / \mathrm{cm}\right)$ groups $(p=0.30)$.

For experiment 2, consecutive breath-holds yielded a significantly larger standard deviation of LV torsion compared to consecutive navigator scans $\left(0.24 \pm 0.10^{\circ} / \mathrm{cm}\right.$ vs $0.18 \pm 0.06 \% \mathrm{~cm}, \quad p=0.02)$. There was a moderate correlation across subjects between the standard deviation of torsion and the standard deviation of end-expiratory position ( $r=0.34, p=0.03$, Fig. 9). The 95\% limits of agreement from the consecutive breath-hold scans and consecutive navigator scans were $\pm 0.74 \% \mathrm{~cm}$ and $\pm 0.56 \% \mathrm{~cm}$, respectively.

\section{Theoretical sample sizes}

The theoretical sample sizes required to detect a $10 \%$ relative difference in peak torsion $\left(\delta=0.34^{\circ} / \mathrm{cm}\right)$ from 

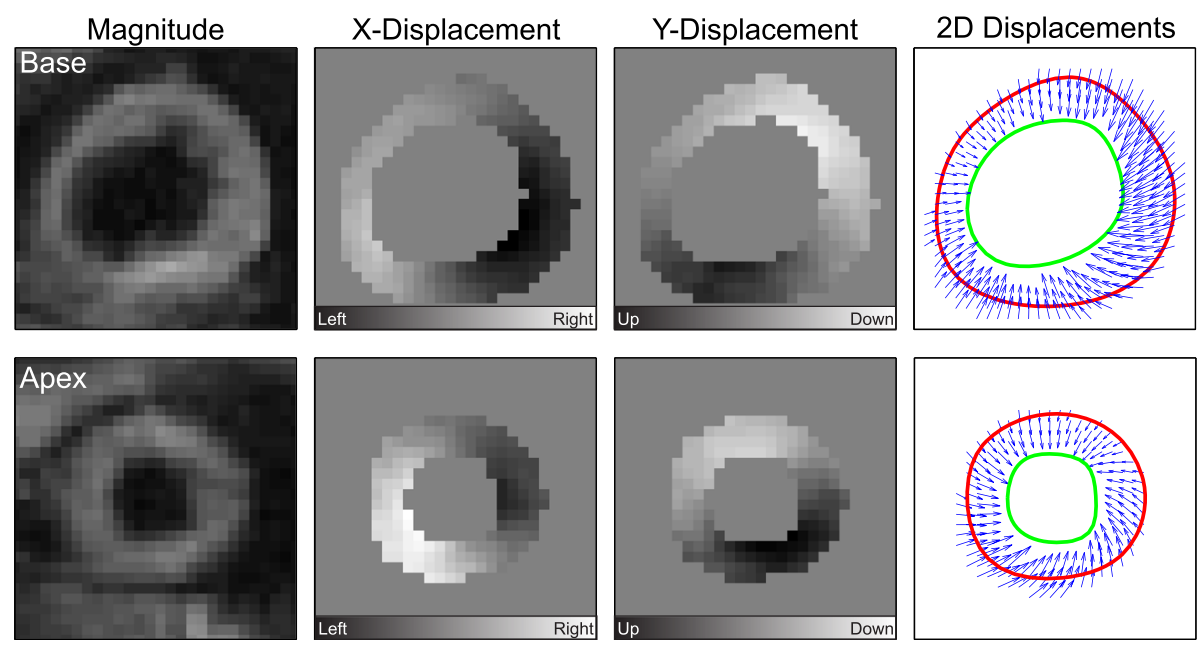

Fig. 7 DENSE images from a representative subject show the relative twist differences between the basal and apical images at end-systole. Twist in the basal region is predominantly in the clockwise direction, while the apex is predominantly counter-clockwise

each experimental protocol are shown in Table 2. From both experiments, using a respiratory navigator with DENSE produced similar sample size estimates $(n=16$ and 15). By comparison, sample sizes based on measurements with variable end-expiratory positions were up to $313 \%$ higher. Additionally, compared to other modalities, using a respiratory navigator with DENSE provided a 80 to $86 \%$ reduction in the required sample size compared to CMR tagging [3], CMR feature tracking [4], and 3D speckle tracking echocardiography [5] (Table 2).

\section{Discussion}

This study explored the effects of inconsistent endexpiratory diaphragm positions on the quantification of LV torsion and how enforcing a consistent end-expiratory position with a respiratory navigator can significantly reduce inter-test variability of measured LV torsion. Our primary findings include 1) using a respiratory navigator with DENSE to enforce a consistent end-expiratory position reduced the variability in measured torsion by $22-57 \%$; 2) this decreased variability reduced the required sample sizes to detect a $10 \%$ relative difference in torsion

Table 1 Mean ( \pm standard deviation) of torsion across the volunteers within each experiment

\begin{tabular}{lll}
\hline Method (experiment) & Torsion $(\% \mathrm{~cm})$ & $p$-value \\
\hline Experiment $1^{\text {a }}$ & & \\
$\quad$ Enforced inconsistent positions & $3.4 \pm 0.4$ & 0.85 \\
$\quad$ Consistent positions with navigator & $3.4 \pm 0.2$ & \\
Experiment 2 & & \\
Breath-holds & $3.6 \pm 0.3$ & 0.32 \\
Consistent positions with navigator & $3.5 \pm 0.2$ & \\
\hline
\end{tabular}

Reported values are from combined group of healthy and patient volunteers from $n=66$ to $n=16$ (from enforced variability to consistent) and $n=26$ to $n=15$ (from natural variability to consistent); 3) the variability of LV torsion due to inconsistent end-expiratory positions had a modest correlation with the variability in end-expiratory positions, such that greater inconsistency in end-expiratory positions was associated with larger errors in measured LV torsion. Regarding inconsistency in end-expiratory positions, within each subject, substantial inconsistency existed with a mean range of $10 \pm 4 \mathrm{~mm}$ and $14 \pm 10 \mathrm{~mm}$ in experiment 1 and 2, respectively, which was similar to that reported previously (7 to $13 \mathrm{~mm}$ ) [6-10].

$\mathrm{LV}$ torsion is an important indicator of cardiac function because it integrates the three-dimensional deformation of the complex myocardial fiber architecture into a single metric $[1,2]$. In many disease states,

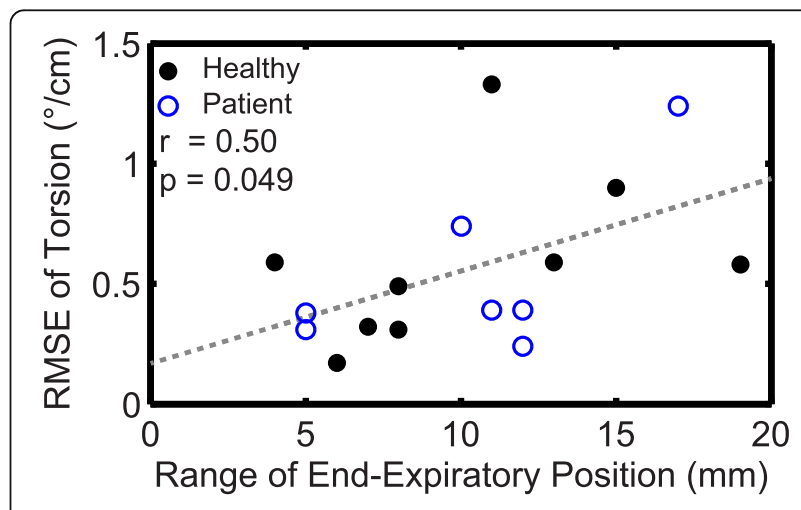

Fig. 8 Variability of torsion due to enforced inconsistent end-expiratory positions versus the subject-specific range of end-expiratory position. There was a moderate positive correlation between RMSE of LV torsion due to inconsistent expiratory positions and the range of end-expiratory position $(r=0.50, p=0.049)$. The dashed gray line illustrates the linear best fit 


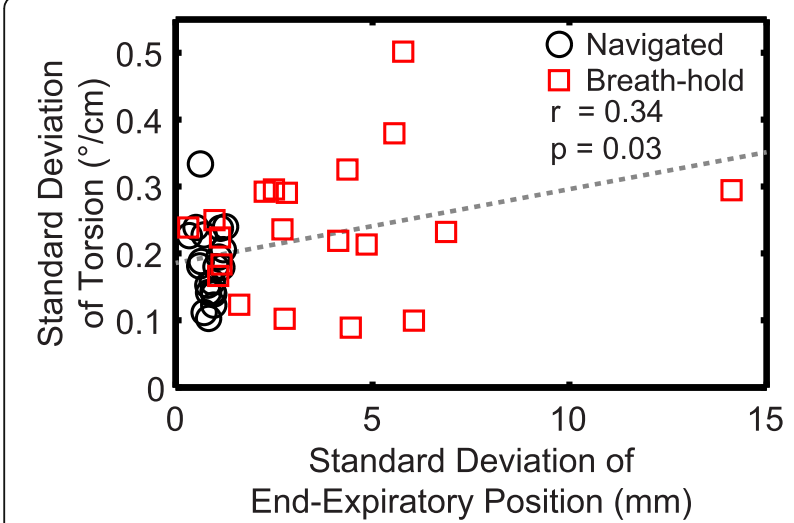

Fig. 9 Variability of torsion due to naturally inconsistent end-expiratory positions versus the standard deviation of end-expiratory position. There was a moderate positive correlation between the standard deviation of LV torsion and the standard deviation of end-expiratory position $(r=0.34, p=0.03)$. The dashed gray line illustrates the linear best fit

small disruptions in normal cardiac geometry-and thus torsion-may precede appreciable changes in global cardiac function. For example, previous studies in mice and canines have reported that changes in torsion precede changes in ejection fraction and volumes in obese animals compared to healthy controls [23, 24]. Previous human studies have reported that LV torsion differs between younger and older populations, and is also reduced in patients with hypertrophic cardiomyopathy, valvular heart disease, previous myocardial infarction, and dilated cardiomyopathy compared to healthy controls [2, 19, 25-28]. Therefore, accurate and reproducible quantification of LV torsion may provide a robust, clinically relevant marker of cardiac health and function.

For LV torsion to be a useful clinical measurement, minimizing the magnitudes and sources of measurement error is important. A previous CMR tagging study reported mean torsion values of $3.4^{\circ} / \mathrm{cm}$ with inter-study

Table 2 Sample sizes required to detect a 10\% relative change in LV torsion calculated using data in this and previous studies

\begin{tabular}{lc}
\hline Method & Sample size (n) \\
\hline Experiment 1 & 66 \\
Enforced inconsistent positions & 16 \\
Consistent positions with navigator & \\
Experiment 2 & 26 \\
Breath-holds & 15 \\
Consistent positions with navigator & \\
Previous Studies & 107 \\
CMR Tagging [3] & 81 \\
CMR Feature Tracking [4] & 80 \\
3D Speckle Tracking [5] & \\
\hline
\end{tabular}

$95 \%$ limits of agreement of $\pm 1.6 \% \mathrm{~cm}$, representing a large percentage of the mean [3]. Previous CMR feature tracking studies have reported inter-test limits of agreement of $\pm 0.9^{\circ} / \mathrm{cm}[4,29]$. Using DENSE CMR, we observed a similar mean of $3.4 \% \mathrm{~cm}$ for all subjects combined in experiment 1 and $3.5 \% \mathrm{~cm}$ in experiment 2 , and smaller inter-test $95 \%$ limits of agreement from the breath-hold scans in experiment $2( \pm 0.74 \% \mathrm{~cm})$. However, the observed inter-test $95 \%$ limits of agreement were considerably smaller when using a respiratory navigator $( \pm 0.6 \% \mathrm{~cm}$ and $\pm 0.56 \% \mathrm{~cm}$ for experiments 1 and 2 , respectively). An important distinction between the present study and previous studies, apart from CMR sequence differences, is control of the end-expiratory position when quantifying the inter-test variability.

From experiment 1 , by comparing the variability of LV torsion inclusive of enforced inconsistent end-expiratory positions $\left(0.56 \pm 0.34^{\circ} / \mathrm{cm}\right)$ to the variability without this inconsistency $\left(0.24 \pm 0.16^{\circ} / \mathrm{cm}\right)$, we determined that using a respiratory navigator to ensure a consistent endexpiratory position reduced the variability in measured LV torsion by $57 \%$. In experiment 2 , using a respiratory navigator reduced the variability in measured LV torsion by $22 \%$ compared to the variability in LV torsion inclusive of naturally inconsistent end-expiratory positions.

In this study, we examined variability in measured LV torsion. A previous CMR study examined the bias in LV twist and circumferential-longitudinal (CL) shear angle between different acquisition techniques, including breath-holds and free-breathing [30]. In agreement with that previous study [30], we did not observe a bias in torsion between breath-hold and navigator-gated scans (Table 1).

To detect a $10 \%$ relative difference in peak LV torsion, experiment 1 found that using DENSE with a respiratory navigator required a sample size of only $n=16$ subjects, which is about $76 \%$ lower than the sample size required when using DENSE without a respiratory navigator $(n=66)$. In experiment 2 , we found similar results where using DENSE with natural respiratory variability required a sample size of 26 compared to using DENSE with a respiratory navigator $(n=15)$. Using a respiratory navigator with DENSE provided a 80 to $86 \%$ reduction in the required sample size compared to CMR tagging [3], CMR feature tracking [4], and 3D speckle tracking echocardiography [5].

These findings have meaningful implications for future CMR-based quantification of LV torsion in the clinical and research settings. First, acquisition of LV torsion data using a respiratory navigator should be employed, where feasible, to minimize variability. This approach is not typical in the majority of published papers reporting torsion and may reduce clinical feasibility of such data acquisition; however, the additional effort appears justified by the 
considerable reduction in variance. If inconsistency in end-expiratory position is not addressed with the data acquisition, then it is important to incorporate effects of inconsistent end-expiratory position into the assessment of the standard error of measurement for LV torsion, which will substantially increase needed sample sizes for research trials or reduce prognostic value for individual subjects.

These results also have important implications for echocardiography. While operators may be able to correct for inconsistency in end-expiratory position by adjusting the position of the probe, it is unlikely that the operator can recreate the exact distance between each short-axis image that was measured from the long-axis image. Because inconsistent end-expiratory positions is a source of measurement variability in measured LV torsion in CMR, the discrepancy in distances may be a source of substantial variability in measured LV torsion in echocardiography.

We used spiral cine DENSE to investigate our hypothesis that inconsistent end-expiratory positions accounts for a significant portion of the variability in measured torsion and that inter-test reproducibility could be improved by using a respiratory navigator. We chose to use spiral cine DENSE to investigate our hypothesis since it allows for simple quantification of mechanics, has good spatial resolution, has good reproducibility, and includes a respiratory navigator, which allows control of the endexpiratory position during image acquisition [11, 31-33]. However, our findings should generalize to all other imaging modalities that use short-axis images to quantify torsion.

\section{Limitations and future directions}

We examined the effects of variable end-expiratory position on LV torsion in a small patient sample. It may be beneficial to examine these results in a larger, more heterogeneous patient sample to determine whether specific diseases affect the results more than others, especially conditions that affect a patient's ability to repeatedly hold his or her breath reproducibly (for example, pulmonary diseases).

Due to the lengthy duration of DENSE breath-holds $(\sim 20 \mathrm{~s})$ and their limitations in breath-holding ability, the breath-hold acquisition protocol was not performed in patients. Based on these factors, we expect that patients would demonstrate higher variability in LV torsion with the breath-hold measures compared to the healthy volunteers we studied. Hence, the potential reduction in mean variability when using the respiratory navigator may in fact be higher than the $22 \%$ we report from the healthy volunteers in experiment 2 . Nevertheless, the reduction in LV torsion variability patients will achieve by using a respiratory navigator will likely fall between the study's reported values of 22 and $57 \%$.

\section{Conclusion}

Using a respiratory navigator to enforce a consistent end-expiratory position during image acquisition can reduce the variability in measured LV torsion by $22-57 \%$. Accounting for inconsistent end-expiratory positions results in favorable inter-test variability and reduces required sample sizes by 80 to $86 \%$ compared to previous studies. Future efforts to measure LV torsion should use a respiratory navigator or similar form of consistent respiratory compensation.

\section{Abbreviations}

CMR: Cardiovascular magnetic resonance; DENSE: Displacement encoding with stimulated echoes; DICOM: Digital imaging and communications in medicine; ECG: Electrocardiogram; LV: Left ventricular (ventricle); RMSE: Root mean squared error

\section{Acknowledgements \\ Not applicable.}

\section{Funding}

This work was supported by a National Institute of Health (NIH) Director's Early Independence Award (DP5 OD-012132), a grant from the National Institute of General Medical Science (P20 GM103527) of the National Institutes of Health, a NIH Ruth L. Kirschstein fellowship (NIH 1F31HL126403), and grant UL1TR000117 from the National Center for Research Resources (NCRR), funded by the Office of the Director, National Institutes of Health and supported by the $\mathrm{NIH}$ Roadmap for Medical Research. The content is solely the responsibility of the authors and does not necessarily represent the official views of the funding sources.

\section{Availability of data and materials \\ The datasets analyzed during the current study are available from the corresponding author on reasonable request.}

\section{Authors' contributions}

$\mathrm{SH}, \mathrm{JS}, \mathrm{CH}, \mathrm{GW}$, and BF conceived the study. SH, GW, KA, and DP assisted with data acquisition. $\mathrm{SH}$ analyzed the data and drafted the initial manuscript. $\mathrm{RC}$ assisted with statistical analysis of the data. $\mathrm{CH}$, JS, and BF helped to interpret the data and draft the manuscript. CH, JS, GW, DP, RC, and BF assisted with analysis and critical revision of the manuscript. All authors read and approved the final manuscript.

\section{Competing interests}

The authors declare that they have no competing interests.

\section{Consent for publication}

Not applicable.

\section{Ethics approval and consent to participate}

The local Institutional Review Board at the University of Kentucky approved the study protocol and all participants provided written informed consent.

\footnotetext{
Author details

'Department of Electrical and Computer Engineering, University of Kentucky, Lexington, KY, USA. ${ }^{2}$ Department of Pediatrics, University of Kentucky, Lexington, KY, USA. ${ }^{3}$ Department of Imaging Science and Innovation, Geisinger Health System, Danville, PA, USA. ${ }^{4}$ Biomedical and Translational Informatics Institute, Geisinger Health System, Danville, PA, USA. ${ }^{5}$ Department of Biomedical Engineering, University of Kentucky, Lexington, KY, USA. ${ }^{6}$ Departments of Biostatistics and Statistics, University of Kentucky, Lexington, KY, USA. 'Departments of Physiology and Medicine, University of Kentucky, Lexington, KY, USA. ${ }^{8}$ Department of Radiology, Geisinger Health System, 100 North Academy Avenue, Danville, PA 17822-4400, USA.
} 
Received: 25 January 2017 Accepted: 8 February 2017 Published online: 01 March 2017

\section{References}

1. Rüssel IK, Götte MJW, Bronzwaer JG, Knaapen P, Walter J, Van Rossum AC. Left ventricular torsion. JACC CardiovasC Imaging. 2011;2:648-55.

2. Götte MJW, Germans T, Rüssel IK, Zwanenburg JJM, Marcus JT, van Rossum AC, van Veldhuisen DJ. Myocardial strain and torsion quantified by cardiovascular magnetic resonance tissue tagging. Studies in normal and impaired left ventricular function. J Am Coll Cardiol. 2006;48:2002-11.

3. Donekal S, Ambale-Venkatesh B, Berkowitz S, Wu CO, Choi EY, Fernandes V, Yan R, Harouni AA, Bluemke DA, Lima JA. Inter-study reproducibility of cardiovascular magnetic resonance tagging. J Cardiovasc Magn Reson. 2013;15:37.

4. Kowallick JT, Morton G, Lamata P, Jogiya R, Kutty S, Lotz J, Hasenfuß G, Nagel E, Chiribiri A, Schuster A. Inter-study reproducibility of left ventricular torsion and torsion rate quantification using MR myocardial feature tracking J Magn Reson Imaging. 2016;43:128-37.

5. Kaku K, Takeuchi M, Tsang W, Takigiku K, Yasukochi S, Patel AR, Mor-Avi V, Lang RM, Otsuji Y. Age-related normal range of left ventricular strain and torsion using three-dimensional speckle-tracking echocardiography. J Am Soc Echocardiogr. 2014;27:55-64.

6. Liu YL, Riederer SJ, Rossman PJ, Grimm RC, Debbins JP, Ehman RL. A monitoring, feedback, and triggering system for reproducible breath-hold MR imaging. Magn Reson Med. 1993;30:507-11.

7. Wang Y, Christy PS, Korosec FR, Alley MT, Grist TM, Polzin JA, Mistretta CA. Coronary MRI with a respiratory feedback monitor: the 2D imaging case. Magn Reson Med. 1995;33:116-21.

8. Taylor AM, Jhooti P, Wiesmann F, Keegan J, Firmin DN, Pennell DJ. MR navigator-echo monitoring of temporal changes in diaphragm position: implications for MR coronary angiography. J Magn Reson Imaging 1997;7:629-36.

9. Holland AE, Goldfarb JW, Edelman RR. Diaphragmatic and cardiac motion during suspended breathing: preliminary experience and implications for breath-hold MR imaging. Radiology. 1998;209:483-9.

10. Fischer RW, Botnar RM, Nehrke K, Boesiger P, Manning WJ, Peters DC. Analysis of residual coronary artery motion for breath hold and navigator approaches using real-time coronary MRI. Magn Reson Med. 2006;55:612-8.

11. Zhong X, Spottiswoode BS, Meyer CH, Kramer CM, Epstein FH. Imaging three-dimensional myocardial mechanics using navigator-gated volumetric spiral cine DENSE MRI. Magn Reson Med. 2010;64:1089-97.

12. Wehner GJ, Suever JD, Haggerty CM, Jing L, Powell DK, Hamlet SM, Grabau JD, Mojsejenko WD, Zhong X, Epstein FH, Fornwalt BK. Validation of in vivo 2D displacements from spiral cine DENSE at 3T. J Cardiovasc Magn Reson. 2015;17:1-11.

13. Wehner GJ, Grabau JD, Suever JD, Haggerty CM, Jing L, Powell DK, Hamlet SM, Vandsburger $\mathrm{MH}$, Zhong X, Fornwalt BK. 2D cine DENSE with low encoding frequencies accurately quantifies cardiac mechanics with improved image characteristics. J Cardiovasc Magn Reson. 2015;17:93.

14. Zhong X, Spottiswoode BS, Cowart EA, Gilson WD, Epstein FH. Selective suppression of artifact-generating echoes in cine DENSE using through-plane dephasing. Magn Reson Med. 2006;56:1126-31.

15. Kim D, Gilson WD, Kramer CM, Epstein FH. Myocardial tissue tracking with two-dimensional cine displacement-encoded MR imaging: development and initial evaluation. Radiology. 2004;230:862-71.

16. Gilliam AD, Suever JD. DENSEanalysis. Retrieved from https://github.com/ denseanalysis/denseanalysis. Accessed 1 Jan 2017.

17. Suever JD, Wehner GJ, Haggerty CM, Jing L, Hamlet SM, Binkley CM, Kramer SP Mattingly AC, Powell DK, Bilchick KC, Epstein FH, Fornwalt BK. Simplified post processing of cine DENSE cardiovascular magnetic resonance for quantification of cardiac mechanics. J Cardiovasc Magn Reson. 2014;16:94.

18. Spottiswoode BS, Zhong X, Hess AT, Kramer CM, Meintjes EM, Mayosi BM, Epstein FH. Tracking myocardial motion from cine DENSE images using spatiotemporal phase unwrapping and temporal fitting. IEEE Trans Med Imaging. 2007;26:15-30.

19. Phan TT, Shivu GN, Abozguia K, Gnanadevan M, Ahmed I, Frenneaux M. Left ventricular torsion and strain patterns in heart failure with normal ejection fraction are similar to age-related changes. Eur J Echocardiogr. 2009;10:793-800.
20. Sorger JM, Wyman BT, Faris OP, Hunter WC, McVeigh ER. Torsion of the left ventricle during pacing with MRI tagging. J Cardiovasc Magn Reson. 2003;5:521-30

21. Hamlet SM, Haggerty CM, Suever JD, Wehner GJ, Grabau JD, Andres KN, Vandsburger MH, Powell DK, Sorrell VL, Fornwalt BK. An interactive videogame designed to improve respiratory navigator efficiency in children undergoing cardiac magnetic resonance. J Cardiovasc Magn Reson. 2016;18:54.

22. Grothues F, Smith GC, Moon JC, Bellenger NG, Collins P, Klein HU, Pennell DJ. Comparison of interstudy reproducibility of cardiovascular magnetic resonance with two-dimensional echocardiography in normal subjects and in patients with heart failure or left ventricular hypertrophy. Am J Cardiol. 2002:90:29-34.

23. Broussard JL, Nelson MD, Kolka CM, Bediako IA, Paszkiewicz RL, Smith L, Szczepaniak EW, Stefanovski D, Szczepaniak LS, Bergman RN. Rapid development of cardiac dysfunction in a canine model of insulin resistance and moderate obesity. Diabetologia. 2016;59:197-207.

24. Kramer SP, Powell DK, Haggerty CM, Binkley CM, Mattingly AC, Cassis LA, Epstein $\mathrm{FH}$, Fornwalt BK. Obesity reduces left ventricular strains, torsion, and synchrony in mouse models: a cine displacement encoding with stimulated echoes (DENSE) cardiovascular magnetic resonance study. J Cardiovasc Magn Reson. 2013;15:109.

25. Oxenham HC, Young AA, Cowan BR, Gentles TL, Occleshaw CJ, Fonseca CG, Doughty RN, Sharpe N. Age-related changes in myocardial relaxation using three-dimensional tagged magnetic resonance imaging. J Cardiovasc Magn Reson. 2003:5:421-30.

26. Young AA, Kramer CM, Ferrari VA, Axel L, Reichek N. Three-dimensional left ventricular deformation in hypertrophic cardiomyopathy. Circulation. 1994;90:854-67.

27. Setser RM, Kasper JM, Lieber ML, Starling RC, McCarthy PM, White RD. Persistent abnormal left ventricular systolic torsion in dilated cardiomyopathy after partial left ventriculectomy. J Thorac Cardiovasc Surg. 2003;126(July):48-55.

28. Garot J, Pascal O, Diébold B, Derumeaux G, Gerber BL, Dubois-Randé J-L, Lima JAC, Guéret P. Alterations of systolic left ventricular twist after acute myocardial infarction. Am J Physiol Heart Circ Physiol. 2002;282:H357-62.

29. Kowallick JT, Lamata P, Hussain ST, Kutty S, Steinmetz M, Sohns JM, Fasshauer M, Staab W, Unterberg-Buchwald C, Bigalke B, Lotz J, Hasenfuß G, Schuster A. Quantification of left ventricular torsion and diastolic recoil using cardiovascular magnetic resonance myocardial feature tracking. PLoS One. 2014;9, e109164.

30. Reyhan ML, Wang Z, Kim HJ, Halnon NJ, Finn JP, Ennis DB. Effect of free-breathing on left ventricular rotational mechanics in healthy subjects and patients with duchenne muscular dystrophy. Magn Reson Med. 2016; 77(2):864-869.

31. Haggerty CM, Kramer SP, Binkley CM, Powell DK, Mattingly AC, Charnigo $R_{\text {, }}$ Epstein FH, Fornwalt BK. Reproducibility of cine displacement encoding with stimulated echoes (DENSE) cardiovascular magnetic resonance for measuring left ventricular strains, torsion, and synchrony in mice. J Cardiovasc Magn Reson. 2013;15:71.

32. Aletras AH, Ding S, Balaban RS, Wen H. DENSE: displacement encoding with stimulated echoes in cardiac functional MRI. J Magn Reson. 1999:137:247-52.

33. Aletras $\mathrm{AH}$, Balaban RS, Wen $\mathrm{H}$. High-resolution strain analysis of the human heart with fast-DENSE. J Magn Reson. 1999:140:41-57.

\section{Submit your next manuscript to BioMed Central and we will help you at every step:}

- We accept pre-submission inquiries

- Our selector tool helps you to find the most relevant journal

- We provide round the clock customer support

- Convenient online submission

- Thorough peer review

- Inclusion in PubMed and all major indexing services

- Maximum visibility for your research

Submit your manuscript at www.biomedcentral.com/submit 